\title{
Partial variables and specificity
}

\author{
Gerhard Jäger \\ University of Potsdam, Institute for Linguistics
}

\begin{abstract}
In this paper I propose a novel analysis of the semantics of specific indefinites. Following standard DRT, I assume that indefinites introduce a free variable into the logical representation, but I assume the the descriptive content of an indefinite DP is interpreted as a precondition of the corresponding variable to denote. Formally this is implemented by an extension of classical predicate logic with partial variables. If a restricted variable is quantified over, the restriction the variable is interpreted as a restriction on the binding quantifier in a syntactically unrestricted way. After an overview over the major existing theories of the scope of indefinites, the central part of the paper is devoted to develop a model-theoretic semantics for this extension of predicate logic. Finally the paper argues that partial variables lends themselves to the analysis of other linguistic phenomena as well. Especially presuppositions can be analyzed as restrictions on variables in a natural way.
\end{abstract}

\section{Introduction}

This article deals with the peculiar scope taking properties of indefinite DPs, which differs massively from other scope bearing elements. The theory that I am going to propose can be seen as a variant of the DRT approach in the version of Heim (1982) according to which the semantic contribution of an indefinite is basically a free variable, while its scope is determined by a non-lexical operation of existential closure. The crucial innovation lies in the treatment of the descriptive material of indefinites. While DRT analyzes it as part of the truth conditions of the local clause, I will argue that it is to be considered as a precondition for the accompanying variable to denote. Existential closure serves a double function in my analysis: it binds a variable, and it turns its definedness conditions into truth conditions.

While the basic idea of my analysis is taken from DRT, it also adopts crucial features of two other current approaches to the scope of indefinites, Tanya Reinhart's choice function approach (Reinhart (1997), see also Winter (1997) and Kratzer (1998)), and the analysis of specificity as a presuppositional phenomenon that was independently proposed by several authors in recent years (among others by Cresti (1995); Reniers (1997); van Geenhoven (1998); Krifka (1998); Yeom (1998); Geurts (1999b)). So my proposal can be seen as an attempt to a synthesis.

The structure of the paper is as follows: in section 2 I will briefly review the empirical phenomena we are going to deal with. Section 3 discusses the most important proposals from the literature. In section 4 the idea of indefiniteness and partial interpretation will be presented, and I will show how this strategy overcomes the main problems that were discussed in the preceding chapters. Section 5 explores the connection between partial variables and presuppostions, and section 6 summarizes the findings. 


\section{Specificity and scope}

The scope of quantifiers is usually clause bounded. This is illustrated in the following examples:

(1) a. If most experiments succeed, Jones will be happy.

b. Jones thanked the student that had conducted most experiments.

In (1a) the quantifier most experiments is embedded in an if-clause. Its scope is restricted to this clause; there is no reading according to which it holds for most experiments $x$ that Jones would be happy if $x$ succeeds. Likewise, the quantifier is embedded in a relative clause in (b) and cannot take scope over the matrix clause.

Most quantifiers behave in a way similar to most experiments in this respect. ${ }^{1}$

This applies to all strong quantifiers, but also to several weak quantifiers, as the following examples illustrate.

(2) a. If every experiment succeeds, Jones will be happy.

b. If at most three experiments succeed, Jones will be happy.

c. If at least three experiments succeed, Jones will be happy.

d. If exactly three experiments succeed, Jones will be happy.

(3) a. Jones thanked the student that had conducted every experiment.

b. Jones criticized the students that had conducted at most three experiments.

c. Jones thanked the students that had conducted at least three experiments.

d. Jones thanked the students that had conducted exactly three experiments.

Singular indefinites like an experiment and plain cardinal quantifiers like three experiments are exceptional in this respect. They can escape scope islands. ${ }^{2}$

(4) a. If an experiment succeeds, Jones will be happy.

b. If three experiments succeed, Jones will be happy.

(4a) has a reading according to which there is a certain experiment whose success would make Jones happy, and likewise for (b), where there are three specific experiments. The same applies to the example with a relative clause. (5a) has a reading where there is a specific experiment such that Jones thanked the students who had conducted it, and analogously for (b).

(5) a. Jones thanked the students that had conducted an experiment.

\footnotetext{
${ }^{1}$ The restrictions on intra-clausal scope are much more complex, and several classes of scope bearing elements have to be distinguished in this respect—see the discussions in Szabolcsi (1997). Farkas and Giannakidou (1996) show that in certain rather restricted configurations, an embedded universal quantifier can take "extra-wide" scope over an indefinite in the matrix clause. Apart from that, definite descriptions and specific indefinites seem to be the only scopal elements that are not clause-bounded.

${ }^{2}$ I will disregard definite descriptions in this article, which also do not obey the usual locality constraints on scope.
} 
b. Jones thanked the students that had conducted three experiments.

These facts where first discussed in Fodor and Sag (1982). They proposed that indefinites are ambiguous between a quantificational and a referential reading. According to them, apparent wide scope indefinites are not scope bearing at all, and the illusion of global scope arises through their referential interpretation.

However, already Farkas (1981) showed that indefinites can take scope outside their local clause without having global scope, and the same point was made again in Abusch (1994).

a. Every writer overheard the rumor that she didn't write a book she wrote. $(\forall>\exists>$ ᄀ) (from Diesing (1992))

b. Every professor got a headache whenever there was a student he hated in class. $(\forall>\exists>$ whenever) (from Abusch (1994))

In the most prominent reading of (6a), the indefinite a book she wrote takes narrow scope with respect to every writer, but wide scope with respect to the rumor and the negation. In (b) $a$ student he hated has scope over the matrix clause even though it is embedded in a when-clause, but it is outscoped by every professor.

These intermediate scope readings do not depend on the presence of a bound pronoun inside the indefinite in question (as the previous examples might suggest). In (7) we have a reading where a boy is outscoped by every town, but outscopes every girl.

(7) In every town, every girl that $a$ boy was in love with married an Albanian. $(\forall>\exists>\forall>$ $\exists$ ) (from Cresti (1995))

It has occasionally been suggested (most recently by Dekker (2002); the idea can be traced back to Hintikka (1986), who confines this approach to indefinites modified by certain though) that these readings are referential despite appearance to the contrary. According to this view, referential indefinites can refer either to individuals or to Skolem functions, and the latter option leads to the illusion of non-global scope. Applied to (7) this means that $a$ boy refers to a specific function $f$ from towns to boys, and the relevant reading can be paraphrased as In every town $t$, every girl that $f(t)$ was in love with married an Albanian. The intermediate scope reading is true if and only if there is a boy-valued function $f$ which makes this paraphrase true.

However, this strategy does not extend to cases where an indefinite with non-local scope is outscoped by a downward monotonic quantifier. Consider

(8) (Some centuries ago, many countries were monarchies, and you could cause a major crisis or even a civil war just by killing the king. Nowadays most countries are republics.) Less than one out of fifty states will cease to function if a particular person is killed.

In the most prominent reading of the last sentence, the indefinite a particular person takes scope over the if-clause, but is outscoped by less than one out of fifty states. Suppose the indefinite has a Skolem function reading. This means that a particular person refers to a Skolem function from states to persons, and the sentence can be paraphrased as Less than one out of 
fifty states $s$ will cease to function if $f(s)$ is killed. Now suppose that each state would cease to function if the head of the secret police gets killed, while the presidents and prime ministers are entirely unimportant. In this scenario, (8) would be false in the intermediate scope reading. However, there is a Skolem function $f$, mapping each state to its president, that would make the above paraphrase true. So the Skolem function reading is certainly not equivalent to the intermediate scope reading. In the above example, intuitions are fairly robust that the intermediate scope reading exists.

The crucial point here is that specificity cannot be reduced to Skolem function readings in the general case. This is not to deny that indefinites can range over Skolem functions, as for instance in

(9) Most professors recommend a certain book, namely their Ph.D. thesis.

The prominent reading of this sentence can be paraphrased as "There is a book valued Skolem function $f$, and most professors $x$ recommend $f(x)$, and $f$ is the function that maps people to their Ph.D. thesis". So Skolem function readings are possible, but this issue is orthogonal to the problem of specificity, and I will not consider it any further in this paper.

From these considerations I conclude that the readings in question are genuine scope readings that cannot be reduced to referentiality. At this point a note on terminology is necessary: indefinites with exceptional scoping behavior are sometimes called "specific", and I will follow this convention. Some authors, however, assume that specificity is more than just non-local scope. It has been suggested that the speaker has to have a particular individual "in mind" which the indefinite in question refers to, or that some other relation of cognitive contact holds between the denotation of the indefinite and some agent. I leave these issues open as orthogonal to the problems I am concerned with in this article. For the time being, when I use the term "specific", it simply means "having non-local scope".

However, the analysis of specific reading of cardinal quantifiers reveals that specificity (even in my broad use of the term) is more complex than just wide scope, as pointed out in Ruys (1992). Consider his

(10) If three relatives of mine die, I'll inherit a fortune.

In the specific reading of three relatives of mine the sentence says that there are three particular relatives of the speaker from whom he would inherit a fortune. According to the standard generalized quantifier treatment, three denotes a relation between two sets which holds iff the intersection of these sets has at least three elements. Using this analysis and giving the quantifier global scope leads to the following semantic representation.

$$
\mid \text { RELATIVE' }^{\prime} \cap \lambda x\left(\operatorname{DIE}^{\prime}(x) \rightarrow \text { INHERIT' }^{\prime}\left(\text { I' }^{\prime}, \text { FORTUNE'}\right)\right) \mid \geq 3
$$

According to (11), the sentence would be true if the speaker has three relatives such that he will inherit a fortune if one of them dies. However, the specific reading actually says that there are three relatives such that the speaker inherits a fortune if each of them dies. This could be represented as 


$$
\begin{aligned}
& \exists X . X \subseteq \text { RELATIVE' } \wedge|X|=3 \wedge \\
& \left(\left(\forall y . y \in X \rightarrow \text { DIE'}^{\prime}(y)\right) \rightarrow \text { INHERIT' }^{\prime}\left(\text { I' }^{\prime}, \text { FORTUNE' }\right)\right)
\end{aligned}
$$

Put in technical terms, simple wide scope for the quantifier amounts to a distributive interpretation, while the specific reading actually requires a collective interpretation. It involves two layers of quantification - a wide scope existential quantification over sets of three relatives of the speaker, and a narrow scope universal quantification over elements of this set. The phenomenon of non-local scope is confined to the existential part.

\section{Solution strategies}

The discussion of the last example shows that a standard scoping mechanism like Quantifier Raising or Cooper storage is not sufficient to account for the peculiarities of specific indefinites. Even if the empirical problem pertaining to the collective/distributive distinction could be overcome within such a framework, we would still be left with the conclusion that there are two versions of the scoping mechanism, one of which is clause bounded and the other unrestricted. This would be a conceptually unsatisfactory state of affairs. Alternatively one might conjecture that the scope of specific indefinites is not derived via the standard scoping mechanism but via some entirely different process. This assumption is only conceptually attractive though if this alternative process can be independently motivated and is not just stipulated for the analysis of specificity.

The literature contains three families of proposals for unbound scoping mechanisms that are applicable to specificity. In this section I will briefly review them and point out their merits as well as their disadvantages.

\subsection{Scope by existential closure}

The literature on Discourse Representation Theory (most notably Heim (1982) and Kamp and Reyle (1993)), contains plenty of evidence that indefinites are non-quantificational. According to this theory, indefinites introduce a free variable into the semantic representation. This variable can be bound by some superordinate unselective binder (like an adverb of quantification). If the variable is not bound by such an operator, a default operation of existential closure applies that amounts to existential quantification over the else free variable.

Existential closure in the sense of DRT is an obvious candidate for a mechanism to assign scope to indefinites. However, it leads to mispredictions if the indefinite has a non-trivial descriptive content. The following example from Reinhart (1995) illustrates this point.

(13) a. If we invite some philosopher, Max will be offended.

b. $\quad \exists x\left(\left(\right.\right.$ PHILOSOPHER' $^{\prime}(x) \wedge$ INVITE' $\left(\right.$ WE$\left.\left.^{\prime}, x\right)\right) \rightarrow$ OFFENDED' $\left(\right.$ MAX'$\left.\left.^{\prime}\right)\right)$

Analyzing (13a) in a DRT-style way without employing any further scoping mechanisms leads to a semantic representation like (13b) for the specific reading of (a), where the existential 
impact of the indefinite some philosopher takes wide scope while the descriptive content remains in the antecedent of the conditional. As already observed in Heim (1982) for an analogous example, (13b) does not represent the truth conditions of the specific reading of (13a). The former is true if there is one non-philosopher, while (13a) in the wide-scope reading requires the existence of a philosopher $x$ with the property that Max will be offended if we invite $x$. Since the existence of the non-philosopher Donald Duck is sufficient to verify (13b) but not (13a), this problem is sometimes called Donald Duck problem in the literature.

\subsection{Indefinites and choice functions}

To overcome this and related problems, several authors proposed to employ choice functions for the analysis of indefinites (see for instance Reinhart (1992), Reinhart (1995), Reinhart (1997), Kratzer (1998), Winter (1997)). To cut a long story short, according to these theories, the semantic counterpart of an indefinite determiner is a variable over a choice function, i.e. a function that maps non-empty sets to one of their elements. This variable is subject to existential closure in a way akin to the treatment of free individual variables in DRT. (13a) would therefore come out as

$$
\exists f\left(C H(f) \wedge\left(\text { INVITE' }^{\prime}\left(\text { WE' }^{\prime}, f\left(\text { PHILOSOPHER' }^{\prime}\right)\right) \rightarrow \text { OFFENDED' }^{\prime}\left(\text { MAX'}^{\prime}\right)\right)\right)
$$

The extension of the predicate constant $C H$ is the set of choice function of type $\langle\langle e, t\rangle, e\rangle$, i.e.

$$
\forall f(C H(f) \leftrightarrow \forall P(\exists x . P(x) \rightarrow P(f(P))))
$$

(14) in fact represents the truth conditions of (13a) in an adequate way. Generally speaking, the choice function approach solves several problems in one stroke. Since it uses unselective binding to assign scope to indefinites, it covers the fact that the scope of indefinites is structurally unrestricted. Second, the choice function mechanism makes sure that the existential impact of an indefinite is not unduly divorced from its descriptive content. Ruys's observation about the double scope of plural specifics can be accommodated into the choice function approach as well in a natural way. (The interested reader is referred to Reinhart (1997) for details.)

On the other hand, the choice function approach faces at least two serious problems. This has been pointed out at several places, for instance in Reniers (1997), Geurts (2000) and Endriss (2001). First, what happens if the extension of the descriptive content of an indefinite is empty? Consider

(15) If you push a certain red button at your computer, the electricity in the whole building will turn off. ${ }^{3}$

Suppose I'm just kidding-your computer is not connected to the power supply. There aren't even any red buttons at your computer. In this case the sentence is certainly false. The choice

\footnotetext{
${ }^{3}$ Adjectives like certain and particular tend to make the specific reading of an indefinite more prominent. I have nothing to say why this is so, but I will use that effect nevertheless whenever appropriate to make the reading I am interested in easier to get.
} 
function approach predicts it to be true though. The extension of red button at your computer is the empty set. There are no restrictions about what kind of value a choice function returns when applied to the empty set.

Let us call this problem the empty set problem.

The second problem arises if the descriptive content of an indefinite contains a pronoun that is bound by some superordinate quantifier. The following example (from Abusch (1994)) can serve to discuss this point.

(16) Every professor ${ }_{i}$ rewarded every student who read some book he ${ }_{i}$ had recommended.

According to the choice function approach, the sentence should have a reading which can be represented as

$$
\begin{aligned}
& \exists f\left(C H ( f ) \wedge \forall x \left(\text { PROFESSOR' } ^ { \prime } ( x ) \rightarrow \forall y \left(\operatorname{STUDENT}^{\prime}(y) \wedge\right.\right.\right. \\
& \left.\left.\left.\operatorname{READ}^{\prime}\left(f\left(\lambda z \cdot \operatorname{BOOK}^{\prime}(z) \wedge \operatorname{RECOMMEND}^{\prime}(x, z)\right)\right) \rightarrow \operatorname{REWARD}^{\prime}(x, y)\right)\right)\right)
\end{aligned}
$$

Suppose two professor, $a$ and $b$, happened to recommend exactly the same books to their students. Then the expressions

$$
\lambda z \cdot \operatorname{BOOK}^{\prime}(z) \wedge \operatorname{RECOMMEND}(a, z)
$$

and

$$
\lambda z \cdot \operatorname{BOOK}^{\prime}(z) \wedge \operatorname{RECOMMEND}^{\prime}(b, z)
$$

denote the same set, and thus the terms

$$
f\left(\lambda z \cdot \operatorname{BOOK}^{\prime}(z) \wedge \text { RECOMMEND' }(a, z)\right)
$$

and

$$
f\left(\lambda z \cdot \operatorname{BOOK}^{\prime}(z) \wedge \operatorname{RECOMMEND}(b, z)\right)
$$

denote the same individual. So the reading that is described in (17) can be paraphrased as follows: Every professor has a favorite book. He recommends this book (possibly along with other books), and he awards students that read his favorite book. Furthermore, if two professors recommend the same books, they have the same favorite book. It might be argued that this reading is actually there but hard to detect, because it is logically stronger than the ordinary narrow-scope reading (17). This is not the case anymore though if we use a downward monotonic quantifier in subject position, as in

(18) At most three girls visited a boy that they fancied.

According to the choice function approach, this sentence should have the reading given in (19a), which — ignoring the empty set problem for the moment—is truth-conditionally equivalent to $(19 b) .^{4}$

\footnotetext{
${ }^{4}$ Chierchia (2001) was presumably the first one to point out that downward monotonic operators are especially problematic for the choice function approach.
} 


$$
\begin{array}{ll}
\text { a. } & \exists f . C H(f) \wedge\left|\lambda x . \operatorname{GIRL}^{\prime}(x) \wedge \operatorname{VISIT}^{\prime}\left(x,\left(f\left(\lambda y . \mathrm{BOY}^{\prime}(y) \wedge \operatorname{FANCY}^{\prime}(x, y)\right)\right)\right)\right| \leq 3 \\
\text { b. } \quad\left|\lambda x . \operatorname{GIRL}^{\prime}(x) \wedge \forall y\left(\operatorname{BOY}^{\prime}(y) \wedge \operatorname{FANCY}^{\prime}(x, y) \rightarrow \operatorname{VISIT}^{\prime}(x, y)\right)\right| \leq 3
\end{array}
$$

So the prediction is that the sentence has a reading that is synonymous to At most three girls visited every boy that they fancied. Intuitions are fairly solid here that such a reading does not exist.

Intuitively, this bound pronoun problem in connection with the choice function approach is similar to to the Donald Duck problem of the unselective binding approach: in both approaches, the interpretation of the descriptive content of an indefinite is separated from its existential impact, while these two semantic components of indefinites always occur in tandem.

This problem is discussed among others by Kratzer (1998) (who attributes the observation to Kai von Fintel and P. Casalegno) and Winter (1997). Again, the literature contains several proposals to circumvent this kind of overgeneration, but none of them handles the interaction of the bound pronouns within an indefinite and a downward entailing quantifier adequately. I will call this problem the bound pronoun problem.

\subsection{Specificity as presupposition accommodation}

Specificity shares several features with presupposition accommodation, and it is thus tempting to unify these two phenomena. This idea has been suggested at several place in the literature. I am aware of the following sources (but there may be more): Cresti (1995), Reniers (1997), van Geenhoven (1998), Krifka (1998), Yeom (1998), and Geurts (1999b).

Consider a classical presupposition trigger like the king. If it occurs in a context where its presupposition "There is a king" is not entailed, this information is accommodated. Accommodation into the global context leads to truth conditions involving a wide scope existential quantification, and likewise intermediate and local accommodation correspond to intermediate and local scope respectively. The mentioned authors agree in the assumption that specific indefinites also trigger an existential presupposition, and their wide/intermediate scope arises via accommodation.

The analogy between (undisputed) presupposition triggers and specifics is in fact striking. To start with, if our example trigger the king occurs within a scope island, its presupposition is nonetheless preferably accommodated at the global level. The preferred reading of (20a) (if uttered in a context where the existence of a king is not established yet) is as in (b).

(20) a. Every Italian watched a film that showed the king in his childhood.

b. $\quad=$ There is a (salient?) king $_{i}$ and every Italian watched a film that showed $\operatorname{him}_{i}$ in his $_{i}$ childhood.

This is paralleled by the preference of specific indefinites for global scope, as illustrated in (21a), which is preferably interpreted as in (b).

(21) a. Every Italian watched a program that showed a certain diva in her youth.

b. $\quad=$ There is a certain $\operatorname{diva}_{i}$ and every Italian watched a program that showed her in $_{i}$ her $_{i}$ youth. 
As discussed in detail in van der Sandt (1992) and Geurts (1999a), the choice between different versions of accommodation is subject to a variety of constraints. Preference for high over low accommodation is the most obvious, but not the strongest one. It is outranked by the so-called "trapping constraint", which is an inviolable constraint. It requires that a bound variable inside a presupposition cannot become unbound via accommodation. Paired with the preference for high accommodation, the prediction is that a presupposition containing a bound variable is preferably accommodated directly below the binder of this variable. This is illustrated in (22). The definite DP her ${ }_{i}$ boyfriend triggers the presupposition she $e_{i}$ has a boyfriend. Since her $r_{i}$ is bound by each girl, accommodation cannot be global, and hence the preferred reading is the intermediate one in (22b).

(22) a. Each girl ${ }_{i}$ claimed that she $_{i}$ had visited her ${ }_{i}$ boyfriend.

b. = Each girl has a boyfriend and claimed that she had visited him.

c. $\quad \neq$ There is a boyfriend that each girl claimed to have visited.

The trapping constraint is also operative in connection with specifics. Global scope is blocked because then $s_{i} e_{i}$ would not be bound by each girl anymore. Accordingly, the intermediate scope reading is preferred.

(23) a. Each girl claimed that she $_{i}$ had visited a certain boy she $e_{i}$ fancied.

b. = Each girl fancies a boy and claimed that she had visited him.

c. $\neq$ There is a boy that each girl claimed to have visited.

Last but not least, accommodation is constrained by a variety of pragmatic constraints pertaining to local and global informativity and consistency. For instance, "local informativity" requires that accommodation must not result in a reading where semantic sub-structures become redundant. Consider (24a). The definite the king of France triggers the presupposition that there is a king of France. Accommodating this at the global level (as indicated in (c)), however, results in a structure where the if-clause of the conditional is redundant. Therefore intermediate accommodation as in (b) is the preferred option.

(24) a. If France is a monarchy, then probably the king of France is bald.

b. = If France is a monarchy, there is a king of France and he is probably bald.

c. $\quad \neq$ There is a king of France, and if France is a monarchy, he is probably bald.

Again the same applies ceteris paribus to specific indefinites.

(25) a. If John is not a single child, probably a certain sibling of him will inherit his house.

b. = If John is not a single child, he has siblings and probably one of them will inherit his house.

c. $\quad \neq$ John has a sibling and if he is not a single child, probably this sibling will inherit his house. 
This approach has a lot of appeal. It relates the non-local behavior of specifics to an independently established mechanism for the derivation of non-local scope, and it offers a principled account of the interaction of specificity with pronoun binding.

On the other hand though, there is at least one conceptual and one technical problem with the equation "specificity = accommodation". First and foremost, presupposition accommodation is usually conceived as a repair strategy, which applies if a presupposition cannot be anchored in the local context where the trigger occurs. Even in a theory like van der Sandt's, where accommodation is a fully legitimate interpretation mechanism, it is only applicable if presupposition binding fails. For instance in (26a), the presupposition "There is a man" that is triggered by the man is preferably bound by the indefinite $a$ man in the if-clause (provided the context of utterance does not supply an alternative antecedent). This results in a reading equivalent to (b). Accommodation would lead to reading (c), but this option is not viable here.

$$
\begin{aligned}
& \text { a. If a man walks, the man talks. } \\
& \text { b. } \quad=\text { If a } \operatorname{man}_{i} \text { walks, he } \text { talks. } \\
& \text { c. } \quad \neq \text { There is a } \operatorname{man}_{k}, \text { and if a } \operatorname{man}_{i} \text { walks, he }{ }_{k} \text { talks. }
\end{aligned}
$$

If specific indefinites were ordinary presupposition triggers, we would expect that they can be bound as well. This is impossible, however. (27a) cannot mean (b).

(27) a. If a man walks, a (certain) man talks.

b. $\neq$ If a $\operatorname{man}_{i}$ walks, he talks.

This problem is perhaps less serious than it seems. Krifka (1998) suggests that a sentence like (27a) cannot have the reading (27b) precisely because (26) has this interpretation. More generally, he assumes that the option to use a definite DP to express a certain meaning blocks the competing option to use a specific indefinite. Van Geenhoven and Geurts suggest that specifics cannot have an anaphoric reading because they are indefinite and therefore subject to Heim's "Novelty Condition".

These two alternative explanations perhaps even compatible-if Krifka is right, it might be possible to reduce the Novelty Condition altogether to a blocking effect. I will leave this issue open here.

The other problem for the specificity-as-accommodation theories has to do with specifics that occur in the nuclear scope of quantificational or conditional constructions. Take the following example (due to van Geenhoven):

(28) a. Every singer ${ }_{i}$ chose a song he ${ }_{i}$ practiced the day before. $\not \equiv$

b. Every singer ${ }_{i}$ chose every song he ${ }_{i}$ practiced the day before.

If the indefinite $a$ song he practiced the day before gets a specific construal, it should be accommodated. Global accommodation is excluded because then the bound pronoun he would end up outside the scope of its binder every singer, and this would violate the trapping constraint. According to van der Sandt's theory, the preferred option should actually be accommodation into 
the restrictor of the quantifier every singer. This would lead to the reading (28b). However, (a) cannot have this reading. The only possible reading is the one where the indefinite has narrow scope.

Krifka argues that accommodation of indefinites into the restrictor of quantificational operators does in fact occur. His example is
a. A green-eyed dog is usually intelligent.
b. USUALLY' [ ] [INTELLIGENT' $(x)$ \{GREENEYED_DOG' $(x)\}$ ]
c. USUALLY' ${ }_{x}\left[\right.$ GREENEYED_DOG' $\left.^{\prime}(x)\right]$ [INTELLIGENT' $\left.(x)\right]$

(29b) sketches the semantic interpretation of (a) (in a DRT-like fashion) before accommodation takes place. Presupposed material is indicated by curly brackets. Accommodation of the presupposition of the nuclear scope into the restrictor plus unselective variable binding leads to the representation in (c), which in fact accurately represents the interpretation of (a). Krifka generally assumes that the generic interpretation of indefinites is a consequence of accommodation into the restrictor of some operator.

This idea is tempting, because it unifies the two "strong" readings of indefinites-specific and generic - in a natural way. However, I think that this proposal is not really viable in the end. First, (28) shows that accommodation into the restrictor is not always possible, even if the overall laws of presupposition accommodation actually seems to enforce it. Quite generally, strong (i.e. specific or generic) indefinites never wind up in the restrictor of an adnominal quantifier. Genericity is confined to adverbial quantification (including the silent GEN operator). Furthermore, genericity is a clause bound phenomenon. This can be seen from the following examples.

a. Bill always invites a girl to a restaurant.

b. $\equiv \forall_{x}\left[\operatorname{GIRL}^{\prime}(x)\right]\left[\operatorname{INVITE}^{\prime}\left(\right.\right.$ BILL' $\left.\left.^{\prime}, x\right)\right]$

(31) a. Bill is always jealous because John invites a girl to a restaurant.

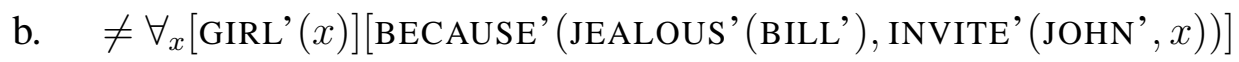

In (30), the adverb always creates a tripartite structure, and the indefinite a girl is generic and supplies the restrictor of the adverb. Accordingly, the truth conditions of (a) are as in (b). In (31), the structure is analogous except that $a$ girl is now embedded in an adjunct clause. Here the indefinite from the embedded clause cannot contribute to the restrictor of the matrix clause. The indefinite can only have the specific (i.e. global scope) or the narrow scope reading. If the generic interpretation of indefinites arose via accommodation, such a sensitivity to syntactic structure would be unexpected. I thus conclude that genericity is an independent phenomenon that can neither be related to specificity nor to presupposition accommodation.

If this is so, then accommodation of specifics into the restrictor of any operators is excluded. Is this an argument against the subsumption of specifics under presuppositions? Not necessarily. The question whether accommodating presuppositions into the restrictor is possible at all has been the topic of long debates (see for instance the discussion in Beaver (2001)). Some examples clearly suggest that it is possible, see for instance (32a). Its preferred reading is (b), the result of (intermediate) accommodation of the presupposition of the nuclear scope of the quantifier into the restrictor. 
(32) a. All Californians quit smoking.

b. All Californians who used to smoke quit smoking.

For other examples, intermediate accommodation is impossible. (33) is due to Beaver:

(33) a. ???Few of the 15 team members and none of the 5 cheerleaders can drive, but every team member will come to the match with her car.

b. Few of the 15 team members and none of the 5 cheerleaders can drive, but every team member that owns a car will come to the match with her car .

c. (???)Few of the 15 team members and none of the 5 cheerleaders can drive, but every team has a car and will come to the match with her car.

Applying intermediate accommodation to (a) yields (b), which is absolutely coherent. However, the only possible reading of (a) is (c) — the result of local accommodation-which is contradictory and thus pragmatically deviant.

The same point can be made with the following sentence, which is a variant of an example due to Hans Kamp:

(34) a. On Saturdays, every German housewife washes her Porsche.

b. Interm. acc: Every German housewife that owns a Porsche washes it on Saturdays.

c. Local acc.: Every German housewife owns a Porsche and washes it on Saturdays.

The (a)-sentence is perceived as unambiguous and entailing that every German housewife owns a Porsche. This corresponds to local accommodation, while intermediate accommodation (sketched in (b)) is no possible reading of (a).

Let us have another look at (32) now. It is a standard assumption that the domain of a quantifier is not the entire universe, but that it is contextually restricted. So an adequate meaning representation of (32a) would be

$$
\forall_{x}\left[\text { CALIFORNIAN' }^{\prime}(x) \wedge C(x)\right]\left[\text { QUIT_SMOKING }^{\prime}(x)\left\{\operatorname{SMOKED}^{\prime}(x)\right\}\right]
$$

The value of the domain variable $C$ has to be contextually supplied. Suppose it is instantiated as used to smoke. This would lead to the representation in (36a). Now the presupposition of the nuclear scope can be bound by material in the restrictor, which leads to the fully specified meaning (36b) for (32).

$$
\begin{aligned}
& \text { a. } \forall_{x}\left[\text { CALIFORNIAN' }^{\prime}(x) \wedge \text { SMOKED' }^{\prime}(x)\right]\left[\text { QUIT_SMOKING }^{\prime}(x)\left\{\operatorname{SMOKED}^{\prime}(x)\right\}\right] \\
& \text { b. } \quad \forall_{x}\left[\text { CALIFORNIAN }^{\prime}(x) \wedge \text { SMOKED' }^{\prime}(x)\right]\left[\text { QUIT_SMOKING' }^{\prime}(x)\right]
\end{aligned}
$$

In general terms, what looks like accommodation into the restrictor can also be analyzed as an instance of presupposition binding, provided the implicit domain restriction of the operator in question supplies an antecedent for the presupposition. It is still surprising that this interpretation strategy is possible in some cases as in (32) while in other cases like (33) or (34) it is impossible. I 
will not resolve this puzzle here, but apparently there are various factors involved here. Arguably the implicit restriction of a domain of quantification from Californians to smoking Californians is more natural from a pragmatic point of view than the shift from German housewives to Porscheowning German housewives. Also, information structure plays an important role. Compare (34) to

\section{Every German housewife washes her Porsche on SATURDAYS}

Here her Porsche is backgrounded, and intermediate accommodation appears to be much easier to get.

Whatever other factors might be involved here, for the present discussion it is only important that strictly speaking there is no operation of accommodation into the restrictor of operators. What looks like it is really an instance of presupposition binding, and specifics cannot be bound due to the Novelty Condition.

Given this, the inability of specifics to be accommodated into the restrictor is entirely regular. So it seems that the analysis of specificity as presupposition accommodation is in fact viable. In the remainder of this article I will develop an analysis of specificity which can be seen as an implementation of this approach. It has the advantage of being compositional, and it offers a simple explanation for the asymmetry between presupposition binding and presupposition accommodation that I argued for above: Binding from the restrictor into the nuclear scope of binary operators is possible, but accommodation into the restrictor is not.

\section{Partial variables}

\subsection{The basic idea}

In this section I will lay out the basic idea of my own proposal. Consider a simple sentence like (38) which contains an indefinite description.

A cup moved.

Following the choice function approach, I assume that the indefinite $a$ cup is an individualdenoting term with the type $e$. Intuitively, it simply refers to some cup. On the other hand, I follow DRT in the assumption that an indefinite is like a variable in predicate logic, i.e. its denotation is underdetermined and has to be fixed by the context. This can straightforwardly be implemented by means of an assignment function. So in the semantic representation $a$ cup is to be translated as a variable (the choice of the name of the variable need not concern us herelet us assume that DPs have referential indices to this effect), but with the side condition that the denotation of this variable has to fall under the extension of the predicate cup. If the local assignment function maps this variable to a non-cup, the indefinite fails to denote.

This idea is implemented by extending the language of first order logic with the notion of a

partial variable. This is a variable that comes with a restriction, i.e. a condition that has to be fulfilled to let the variable have an interpretation. The syntactic convention is simple: 
Definition 1 (Partial variables - syntax) If $x$ is a variable and $\varphi$ is a formula, then $[x \mid \varphi]$ is a term (called a partial variable).

Partial variables are interpreted via assignment functions, provided the restriction is fulfilled.

\section{Definition 2 (Partial variables - semantics (first version))}

$$
\|[x \mid \varphi]\|_{g}=\left\{\begin{array}{l}
g(x) \text { iff }\|\varphi\|_{g}=1 \\
\text { undefined else }
\end{array}\right.
$$

Using partial variables thus leads to a semantics with denotation gaps. Unless otherwise stated, the meaning of a complex expression is undefined if one of its subexpressions is undefined. Definedness conditions are thus inherited from partial variables to expressions containing them. There is one exception to this rule-existential quantification. ${ }^{5}$

\section{Definition 3 (Semantics of complex expressions (first version))}

$$
\begin{gathered}
\|\exists x \varphi\|_{g}=\left\{\begin{array}{l}
1 \text { iff for some } a:\|\varphi\|_{g[a / x]}=1 \\
0 \text { else }
\end{array}\right. \\
\|\forall x \varphi\|_{g}=\left\{\begin{array}{l}
1 \text { iff for all } a:\|\varphi\|_{g[a / x]}=1 \\
0 \text { else }
\end{array}\right.
\end{gathered}
$$

If $\alpha$ is a complex expression but not a partial variable or a quantified formula, and the immediate subexpressions of $\alpha$ are $\beta_{1}, \cdots, \beta_{n}$, then $\|\alpha\|_{g}$ is defined iff $\left\|\beta_{i}\right\|_{g}$ is defined for all $i$ with $1 \leq i \leq$ $n$. If it is defined, it is determined by the rules of standard predicate logic.

The clauses for quantification are identical to their classical counterpart, but they are conceptually different. If the formula in the scope of existential quantifier is false or undefined for all values of the quantified variable, the formula as a whole is just false. This contrasts with other operators, where undefinedness is passed on from sub-constituents to super-constituents.

Let us apply these rules to the simple example from above. In the mapping from English sentences to LFs, I follow largely the version of DRT that is developed in chapter 2 of Heim (1982). The main difference is that indefinites are translated as partial variables rather than as open formulas. These variables are bound by a default operation of existential closure. Following the spirit of Reinhart's version of the choice function approach, I assume that existential closure is selective and can freely apply at every sentential level or at the text level. Put differently, I admit the adjunction of existential quantifiers at every S-node. I represent meanings by means of first order formulas, but the back-and-forth translations between LFs and formulas should be obvious.
a. A cup moved.
b. MOVE' $\left(\left[x \mid \operatorname{CUP}^{\prime}(x)\right]\right)$

\footnotetext{
${ }^{5}$ The universal quantifier is defined in terms of the existential quantifier and negation in the usual way.
} 


$$
\begin{aligned}
& \text { c. } \quad \exists x \cdot \operatorname{MOVE}^{\prime}\left(\left[x \mid \operatorname{CUP}^{\prime}(x)\right]\right) \\
& \text { d. } \| \text { MOVE }^{\prime}\left(\left[x \mid \text { CUP' }^{\prime}(x)\right]\right) \|_{g}=\left\{\begin{array}{l}
1 \text { iff } g(x) \in \| \text { CUP' }^{\prime}\left\|_{g} \cap\right\| \text { MOVE'}^{\prime} \|_{g} \\
0 \text { iff } g(x) \in \| \text { CUP' }^{\prime}\left\|_{g}-\right\| \text { MOVE' }^{\prime} \|_{g} \\
\text { undefined iff } g(x) \notin \| \text { CUP' }^{\prime} \|_{g}
\end{array}\right. \\
& \text { e. }\left\|\exists x \cdot \operatorname{MOVE}^{\prime}\left(\left[x \mid \operatorname{CUP}^{\prime}(x)\right]\right)\right\|=\left\{\begin{array}{l}
1 \text { iff }\left\|\operatorname{CUP}^{\prime}\right\|_{g} \cap \| \text { MOVE'}^{\prime} \|_{g} \neq \emptyset \\
0 \text { else }
\end{array}\right.
\end{aligned}
$$

(39a) is translated as (b), where the verb corresponds to a predicate and the indefinite subject to a partial variable. The NP-part of the indefinite is mapped to the restriction of the variable. Applying existential closure yields (b). The interpretation of (b) is given in (d). Function application passes the definedness conditions of its argument on to the entire expression, so (b) only has an interpretation if the partial variable in it denotes, i.e. if $x$ is mapped to a cup. If this is the case, the truth conditions of (b) are straightforward. Applying existential quantification to this (b) leads to (c) with the interpretation (d). Here undefinedness and falsity are collapsed to falsity, and the bivalent truth conditions are as desired.

Suppose (b) is interpreted in a model where there are no cups. Then the interpretation is undefined regardless of the value of $x$ under $g$. Accordingly, (c) would come out as false under each assignment. There is thus no empty set problem under this approach-existential quantification over an empty set leads to falsity.

Let us move on to a more complex example. In (40a), an indefinite is embedded in an ifclause. (The specific reading is perhaps not all that natural here-try to imagine that there is one psychic cup which reacts to ghosts, while the other cups on the table are just ordinary china.)

(40) a. If a cup moved the ghost is present.

b. $\quad \exists x\left(\operatorname{MOVE}^{\prime}\left(\left[x \mid \mathrm{CUP}^{\prime}(x)\right]\right) \rightarrow \mathrm{GHIP}^{\prime}\right)$

c. $\| \operatorname{MOVE}{ }^{\prime}\left(\left[x \mid\right.\right.$ CUP' $\left.\left.^{\prime}(x)\right]\right) \rightarrow$ GHIP' $^{\prime} \|_{g}=$

$\left\{\begin{array}{l}1 \text { iff } g(x) \in \| \text { CUP' }^{\prime} \|_{g} \&\left(g(x) \in \| \text { MOVE' }\left\|_{g} \Rightarrow\right\| \text { GHIP' }^{\prime} \|_{g}=1\right) \\ 0 \text { iff } g(x) \in \| \text { CUP' }\left\|_{g} \cap\right\| \text { MOVE' }\left\|_{g} \&\right\| \text { GHIP' }_{g} \|_{g}=0 \\ \text { undefined iff } g(x) \notin \| \text { CUP' }^{\prime} \|_{g}\end{array}\right.$

d. $\quad\|(b)\|_{g}= \begin{cases}1 & \text { iff for some } a \in \| \text { CUP' } \|_{g}: \\ & a \in \| \text { MOVE' }\left\|_{g} \Rightarrow\right\| \text { GHIP' }^{\prime} \|_{g}=1 \\ 0 & \text { else }\end{cases}$

e. $\quad \exists x\left(\operatorname{CUP}^{\prime}(x) \wedge\left(\operatorname{MOVE}^{\prime}(x) \rightarrow \operatorname{GHIP}^{\prime}\right)\right)$

Existential closure could be applied either to the if-clause or to the matrix clause. We are interested in the specific reading, which corresponds to the latter option. The translation of (a) under this reading is $(b)$.

The definedness condition of the partial variable is passed on to the translation of the local clause as above, and further on to the entire implication corresponding to the conditional construction. The interpretation of the implication is thus as in (c). Applying existential quantification leads to (d). (40b) thus has the same truth conditions as the wide scope restricted existential quantification in (e). However, this effect has been achieved without syntactically moving the restrictor from its in-situ position. This shows that analyzing indefinites as partial variables avoids 
the Donald Duck problem but nevertheless predicts that the scope of indefinites is syntactically unrestricted.

\subsection{Stacked variables}

The present approach overcomes the empty set problem and the Donald Duck problem, but how about the bound pronoun problem? A critical example would be (41a). Under wide scope existential closure, its translation is as in (b).

(41) a. Every girl visited a boy she fancied.

b. $\quad \exists y \forall x \cdot \operatorname{GIRL}^{\prime}(x) \rightarrow \operatorname{ViSIT}^{\prime}\left(x,\left[y \mid\right.\right.$ BOY $\left.\left.^{\prime}(y) \wedge \operatorname{FANCY}^{\prime}(x, y)\right]\right)$

What are the truth conditions of (41b)? Let us start with the consequent of the implication.

$$
\begin{aligned}
& \|_{\text {VISIT }}\left(x,\left[y \mid \text { BOY }^{\prime}(y) \wedge \text { FANCY }^{\prime}(x, y)\right]\right) \|_{g} \\
& \quad=\left\{\begin{array}{l}
1 \quad \text { iff } g(y) \in \| \text { BOY }^{\prime}\left\|_{g} \&\langle g(x), g(y)\rangle \in\right\| \text { FANCY }^{\prime}\left\|_{g} \cap\right\| \text { VISIT' }^{\prime} \|_{g} \\
0 \quad \text { iff } g(y) \in \| \text { BOY }^{\prime}\left\|_{g} \&\langle g(x), g(y)\rangle \in\right\| \text { FANCY'}^{\prime}\left\|_{g} \backslash\right\| \text { VISIT }^{\prime} \|_{g} \\
\text { undefined else }
\end{array}\right.
\end{aligned}
$$

Accordingly, the implication as a whole has the interpretation

$$
\begin{gathered}
\left\|\operatorname{GIRL}^{\prime}(x) \rightarrow \operatorname{VISIT}^{\prime}\left(x,\left[y \mid \mathrm{BOY}^{\prime}(y) \wedge \mathrm{FANCY}^{\prime}(x, y)\right]\right)\right\|_{g} \\
=\left\{\begin{array}{c}
1 \quad \text { iff } \quad g(y) \in\left\|\mathrm{BOY}^{\prime}\right\|_{g} \&\langle g(x), g(y)\rangle \in\left\|\mathrm{FANCY}^{\prime}\right\|_{g} \& \\
g(x) \in\left(\left\|\mathrm{GIRL}^{\prime}\right\|_{g} \Rightarrow\langle g(x), g(y)\rangle \in\|\mathrm{VISIT}\|_{g}\right) \\
\left.0 \quad \text { iff } \begin{array}{l}
g(y) \in\left\|\mathrm{BOY}^{\prime}\right\|_{g} \&\langle g(x), g(y)\rangle \in\left\|\mathrm{FANCY}^{\prime}\right\|_{g} \& \\
\left(g(x) \in\left\|\mathrm{GIRL}^{\prime}\right\|_{g} \&\langle g(x), g(y)\rangle \notin \| \mathrm{VISIT}\right.
\end{array} \|_{g}\right) \\
\text { undefined else }
\end{array}\right.
\end{gathered}
$$

Applying universal quantification brings us back into bivalent semantics.

$$
\begin{aligned}
& \left\|\forall x . G I R L^{\prime}(x) \rightarrow \operatorname{VISIT}^{\prime}\left(x,\left[y \mid \mathrm{BOY}^{\prime}(y) \wedge \mathrm{FANCY}^{\prime}(x, y)\right]\right)\right\|_{g} \\
& =\left\{\begin{array}{cc}
1 & \text { iff } \quad \text { for all } a: g(y) \in\left\|\mathrm{BOY}^{\prime}\right\|_{g} \&\langle a, g(y)\rangle \in\left\|\mathrm{FANCY}^{\prime}\right\|_{g} \& \\
0 & \text { else }
\end{array}\right.
\end{aligned}
$$

After existentially binding $y$ we get

$$
\begin{aligned}
& \left\|\exists y \forall x\left(\operatorname{GIRL}^{\prime}(x) \rightarrow \operatorname{VISIT}^{\prime}\left(x,\left[y \mid \mathrm{BOY}^{\prime}(y) \wedge \operatorname{FANCY}^{\prime}(x, y)\right]\right)\right)\right\|_{g}
\end{aligned}
$$

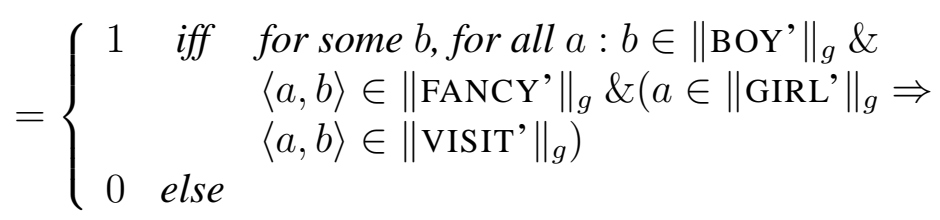


These are the truth conditions of the classical formula (46a), which is equivalent to (b).

$$
\begin{aligned}
& \text { a. } \quad \exists y \forall x \cdot \text { BOY }^{\prime}(y) \wedge \operatorname{FANCY}^{\prime}(x, y) \wedge\left(\operatorname{GIRL}^{\prime}(x) \rightarrow \operatorname{VISIT}^{\prime}(x, y)\right) \\
& \text { b. } \quad \exists y \cdot \operatorname{BOY}^{\prime}(y) \wedge \forall x \text { FANCY }^{\prime}(x, y) \wedge \forall x\left(\operatorname{GIRL}^{\prime}(x) \rightarrow \operatorname{VISIT}^{\prime}(x, y)\right)
\end{aligned}
$$

It is obvious that this has nothing to do with the intuitive truth conditions of (41). For (46) to be true, there has to be some boy who is fancied by everybody, while no such thing is entailed by (41).

The problem here is that the variable $x$ that occurs in the restriction of $y$ is bound at a position where $y$ is still free. However, this occurrence of $x$ depends on $y$ because the former is part of the restriction of the latter. The problem arises because a dependent variable is bound, which ought to be illicit.

I will first explain in syntactic terms what goes wrong here, before I present a revised semantics of first order logic with partial variables. Basically, existential quantification turns a definedness condition of the bound variable into an ordinary restriction of the existential quantifier. This can be formulated as a syntactic transformation rule:

$$
\exists x \varphi([x \mid \psi]) \mapsto \exists x . \psi \wedge \varphi(x)
$$

If the descriptive part of a specific indefinite always has the same scope as the corresponding existential quantifier (i.e. if there is no version of the Donald Duck problem whatsoever), this rule must be meaning-preserving.

Now reconsider formula (41b):

$$
\exists y \forall x \cdot \operatorname{GIRL}^{\prime}(x) \rightarrow \operatorname{VISIT}^{\prime}\left(x,\left[y \mid \mathrm{BOY}^{\prime}(y) \wedge \operatorname{FANCY}^{\prime}(x, y)\right]\right)
$$

Applying the transformation rule would give us

$$
\exists y \cdot \text { BOY }^{\prime}(y) \wedge \text { FANCY }^{\prime}(x, y) \wedge \forall x . \operatorname{GIRL}^{\prime}(x) \rightarrow \operatorname{VISIT}^{\prime}(x, y)
$$

Here the first occurrence of $x$ is free. This does in fact correspond to a possible reading of (41), the one where the pronoun she is free. Given the present semantics, this transformation is not meaning preserving though because there is a variable occurrence that is bound before and free after applying the transformation. To make the transformation meaning preserving, we thus have to ensure that a variable occurrence $x$ that occurs inside the restriction of some free variable $y$ cannot be bound by a quantifier outside the restriction of the same occurrence of $y$.

We could enforce this by requiring that quantifiers only bind independent variable occurrences. This would not suffice though. After applying the transformation, dependent variable occurrence become independent and thus free to be bound. If this is a meaning preserving operation, it must be possible to bind bound variable occurrences after all. The crucial restriction is that the scope relation between the binders and the dependency relation between the bound elements must be inverse from each other. Schematically, the following structure would represent a possible binding configuration: 


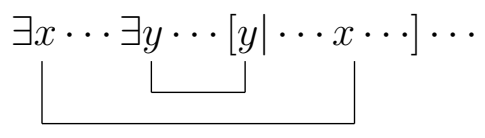

Here the binding relations are nested. Crossing binding relations ought to be illicit:

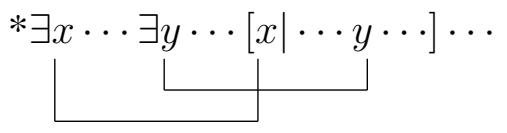

Likewise, binding into the restriction of a free variable is bad:

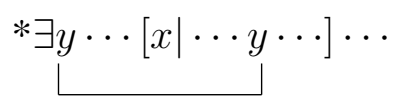

How can the illicit configurations be avoided? I will use a procedural metaphor. When constructing a formula bottom-up, variable occurrences are arranged in stacks. A single variable creates a new stack with this variable as the only element. If a variable occurrence $x$ is put into the restriction of a variable occurrence $y, y$ is pushed on top of the stack containing $x$. This may apply recursively, so that stacks may be arbitrarily large. Binding a variable with a quantifier has the effect of popping elements from stacks. The quantifier $\exists x$ removes all occurrences of $x$ that are top elements of a stack from their stacks. Now all variable occurrences that directly depend on a top-level occurrence of $x$ become top-level elements themselves and are thus free to be bound. Crucially, variable occurrences that are not top-level cannot be bound.

The technical implementation of this goes as follows. Assignment functions are not just applied to single variables, but to non-empty sequences of variables - the formal counterpart of variable stacks. If the variable $x$ occurs freely and independently in a formula, its interpretation under some assignment $g$ is just $g(x) . x$ might as well occur within the restriction of another variable $y$, as for instance in $[y \mid R(y, x)]$. Then the interpretation of the occurrence of $x$ is $g(x y)$. This system works recursively. If you have an expression like $[z \mid S(z,[y \mid R(y, x)])]$, then the interpretation of this occurrence of $x$ under $g$ would be $g(z y x)$, etc. So assignment functions do not evaluate single variable occurrences but entire stacks.

The interpretation rule for partial variables has to be changed accordingly. I use the following notational convention: if $g$ is an assignment and $x$ a variable, then $g^{x}$ is the assignment with the property that for all non-empty sequences $\vec{v}$

$$
g^{x}(\vec{v})=g(x \vec{v})
$$

This is the semantic implementation of pushing $x$ on the stack $v$. This operation is needed to interpret partial variables:

\section{Definition 4 (Partial variables - semantics (revised version))}

$$
\|[x \mid \varphi]\|_{g}=\left\{\begin{array}{l}
g(x) \text { iff }\|\varphi\|_{g^{x}}=1 \\
\text { undefined else }
\end{array}\right.
$$


In linguistic applications, the restriction $\varphi$ of a variable occurrence $x$ should properly restrict the denotation of $x$. So $x$ should occur freely in $\varphi$. However, free occurrences of $x$ in $\varphi$ are now one level lower in the stack than the occurrence of $x$ they restrict. To avoid this side effect, I assume that pushing $x$ on a stack where $x$ is already the top element has no effect on the stack.

Quantification turns the restriction of the bound variable into a part of the truth conditions. This means that variables that depend on the quantified variable are turned into independent variables. Hence we have to adjust the definition of quantification as well.

We need two more auxiliary notions:

- $g[a / x]$ is the assignment which is exactly like $g$ except that $g\left(x^{n}\right)=a .^{6}$ This ensures that two occurrences of $x$ where the first directly depends on the second refer to the same object.

- $g\{x\}$ is the assignment which is exactly like $g$ except that for all non-empty sequences $\vec{v}$ that start with a variable $\neq x$ :

$$
g\{x\}\left(x^{n} \vec{v}\right)=g(\vec{v})
$$

This implements the notion of popping all top-level occurrences of $x$ from a stack.

Quantification pops top-level elements from stacks and quantifies over their denotation:

\section{Definition 5 (Semantics of Quantification (revised version))}

$$
\begin{aligned}
& \|\exists x \varphi\|_{g}=\left\{\begin{array}{l}
1 \text { iff for some } a:\|\varphi\|_{g[a / x]\{x\}}=1 \\
0 \text { else }
\end{array}\right. \\
& \|\forall x \varphi\|_{g}=\left\{\begin{array}{l}
1 \text { iff for all } a:\|\varphi\|_{g[a / x]\{x\}}=1 \\
0 \text { else }
\end{array}\right.
\end{aligned}
$$

It might be helpful to go through the interpretation procedure for (41) under the revised interpretation to see the difference.

The formula to be interpreted is still

$$
\exists y \forall x . \operatorname{GIRL}^{\prime}(x) \rightarrow \operatorname{VISIT}^{\prime}\left(x,\left[y \mid \operatorname{BOY}^{\prime}(y) \wedge \operatorname{FANCY}^{\prime}(x, y)\right]\right)
$$

Skipping over some intermediate steps that ought to be obvious, the interpretation of the implication is

$$
\begin{aligned}
& \left\|\operatorname{GIRL}^{\prime}(x) \rightarrow \operatorname{ViSIT}^{\prime}\left(x,\left[y \mid \operatorname{BOY}^{\prime}(y) \wedge \operatorname{FANCY}^{\prime}(x, y)\right]\right)\right\|_{g} \\
& =\left\{\begin{array}{rrr}
1 & \text { iff } \quad & g^{y}(y) \in\left\|\mathrm{BOY}^{\prime}\right\|_{g} \&\left\langle g^{y}(x), g^{y}(y)\right\rangle \in\left\|\mathrm{FANCY}^{\prime}\right\|_{g} \& \\
& \left(g(x) \in\left\|\mathrm{GIRL}^{\prime}\right\|_{g} \Rightarrow\langle g(x), g(y)\rangle \in\left\|\mathrm{VISIT}^{\prime}\right\|_{g}\right) \\
0 \quad \text { iff } \quad & g^{y}(y) \in\left\|\mathrm{BOY}^{\prime}\right\|_{g} \&\left\langle g^{y}(x), g^{y}(y)\right\rangle \in\left\|\mathrm{FANCY}^{\prime}\right\|_{g} \& \\
& \left(g(x) \in\left\|\mathrm{GIRL}^{\prime}\right\|_{g} \&\langle g(x), g(y)\rangle \notin\left\|\mathrm{VISIT}^{\prime}\right\|_{g}\right) \\
\text { undefined else } &
\end{array}\right.
\end{aligned}
$$

\footnotetext{
${ }^{6}$ The notation $x^{n}$ stands for a non-empty sequence of $x$.
} 
Universal quantification over $x$ yields

$$
\begin{aligned}
& \| \forall x . \text { GIRL' }^{\prime}(x) \rightarrow \operatorname{VISIT}^{\prime}\left(x,\left[y \mid \text { BOY }^{\prime}(y) \wedge \text { FANCY }^{\prime}(x, y)\right]\right) \|_{g}
\end{aligned}
$$

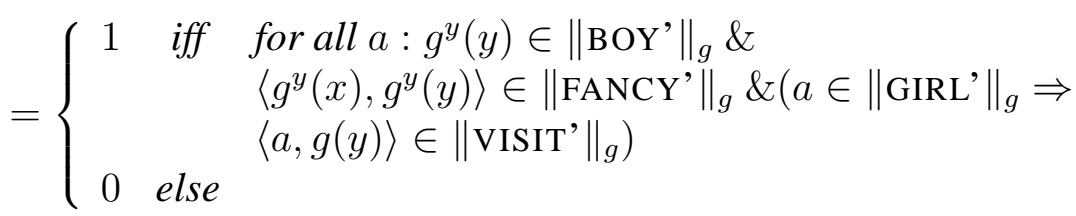

Here the shift from the assignment function $g[a / x]$ to $g[a / x]\{x\}$ has no effect since no variables depend on $x$. This is different for $y$ :

$$
\begin{aligned}
& \left\|\exists y \forall x . \operatorname{GIRL}^{\prime}(x) \rightarrow \operatorname{VISIT}^{\prime}\left(x,\left[y \mid \mathrm{BOY}^{\prime}(y) \wedge \operatorname{FANCY}^{\prime}(x, y)\right]\right)\right\|_{g} \\
& = \begin{cases}1 \quad \text { iff } \quad & \text { for some } b, \text { for all } a: b \in\left\|\mathrm{BOY}^{\prime}\right\|_{g[b / y]\{y\}^{y}} \& \\
& \left\langle g[b / y]\{y\}^{y}(x), b\right\rangle \in\left\|\mathrm{FANCY}^{\prime}\right\|_{g[b / y]\{y\}^{y}} \& \\
& \left(a \in\left\|\mathrm{GIRL}^{\prime}\right\|_{g[b / y]\{y\}} \Rightarrow\langle a, b\rangle \in\left\|\operatorname{VISIT}^{\prime}\right\|_{g[b / y]\{y\}^{y}}\right)\end{cases} \\
& \hline \begin{array}{lll}
\text { else } &
\end{array}
\end{aligned}
$$

Going from $g$ to $g^{y}$ amounts to pushing one occurrence of $y$ to every stack, while going from $g$ to $g\{y\}$ amounts to removing all leading occurrences of $y$ from every stack. Taken together this means that $g\{y\}^{y}=g\{y\}$, hence we can simplify to

$$
\begin{aligned}
& \| \exists y \forall x \text {.GIRL' }(x) \rightarrow \operatorname{VISIT}^{\prime}\left(x,\left[y \mid \text { BOY }^{\prime}(y) \wedge \text { FANCY }^{\prime}(x, y)\right]\right) \|_{g} \\
& =\left\{\begin{array}{rrr}
1 \quad \text { iff } \quad & \text { for some } b, \text { for all } a: b \in \| \text { BOY' }^{\prime} \|_{g} \& \\
& \langle g(x), b\rangle \in \| \text { FANCY' } \|_{g} \& \\
& \left(a \in \| \text { GIRL' }_{g} \Rightarrow\langle a, b\rangle \in \| \text { VISIT' }_{g} \|_{g}\right) \\
0 \quad \text { else } &
\end{array}\right.
\end{aligned}
$$

These are the truth conditions of the classical formula

$$
\exists y \cdot \operatorname{BOY}^{\prime}(y) \wedge \operatorname{FANCY}^{\prime}(x, y) \wedge \forall x . \operatorname{GIRL}^{\prime}(x) \rightarrow \operatorname{VISIT}^{\prime}(x, y)
$$

This is exactly the result of the syntactic transformation briefly considered above. It represents the (possible) reading where the indefinite is specific and the pronoun in its descriptive part is free. It is NOT possible anymore to derive a reading where the indefinite has wide scope but the pronoun is bound by the universal quantifier. So the bound pronoun problem is solved as well. 


\subsection{One variable at a time}

Still, the theory in its present shape makes wrong predictions when several indefinites interact.

(53) a. It is not the case that a certain boy met a girl.

b. $\quad \exists x \neg\left(\exists y \operatorname{MEET}^{\prime}\left(\left[x \mid \operatorname{BOY}^{\prime}(x)\right],\left[y \mid \operatorname{GIRL}^{\prime}(y)\right]\right)\right.$

In (53) we have two indefinites, and we are interested in the reading where the first is existentially bound with wide and the second with narrow scope. The interpretation of the atomic formula in the scope of the second quantifier is straightforward:

$$
\begin{aligned}
& \| \operatorname{MEET}^{\prime}\left(\left[x \mid \text { BOY'}^{\prime}(x)\right],\left[y \mid \operatorname{GIRL}^{\prime}(y)\right]\right) \|_{g} \\
& =\left\{\begin{aligned}
1 \quad \text { iff } & g^{x}(x) \in\left\|\mathrm{BOY}^{\prime}\right\|_{g} \& g^{y}(y) \in\left\|\mathrm{GIRL}^{\prime}\right\|_{g} \& \\
& \langle g(x), g(y)\rangle \in\left\|\mathrm{MEET}^{\prime}\right\|_{g} \\
0 \quad \text { iff } \quad & g^{x}(x) \in\left\|\mathrm{BOY}^{\prime}\right\|_{g} \& g^{y}(y) \in\left\|\mathrm{GIRL}^{\prime}\right\|_{g} \& \\
& \langle g(x), g(y)\rangle \notin\left\|\mathrm{MEET}^{\prime}\right\|_{g} \\
\text { undefined else } &
\end{aligned}\right.
\end{aligned}
$$

Applying existential quantification gives us

$$
\begin{aligned}
& \| \exists y \operatorname{MEET}^{\prime}\left(\left[x \mid \text { BOY }^{\prime}(x)\right],\left[y \mid \operatorname{GIRL}^{\prime}(y)\right]\right) \|_{g} \\
& =\left\{\begin{array}{ccc}
1 & \text { iff } \quad \text { for some } a: g^{x}(x) \in\left\|\mathrm{BOY}^{\prime}\right\|_{g} \& a \in\left\|\mathrm{GIRL}^{\prime}\right\|_{g} \& \\
& \langle g(x), a\rangle \in\left\|\mathrm{MEET}^{\prime}\right\|_{g}
\end{array}\right.
\end{aligned}
$$

Existential quantification turns definedness conditions into truth conditions, and it does so in a non-discriminating way. It does not distinguish to which partial variable the different parts of the definedness conditions belong. This has disastrous consequences here. Continuing with negation and a second existential quantification, we get

$$
\begin{aligned}
& \| \exists x \neg \exists y \operatorname{MEET}^{\prime}\left(\left[x \mid \text { BOY }^{\prime}(x)\right],\left[y \mid \mathrm{GIRL}^{\prime}(y)\right]\right) \|_{g}
\end{aligned}
$$

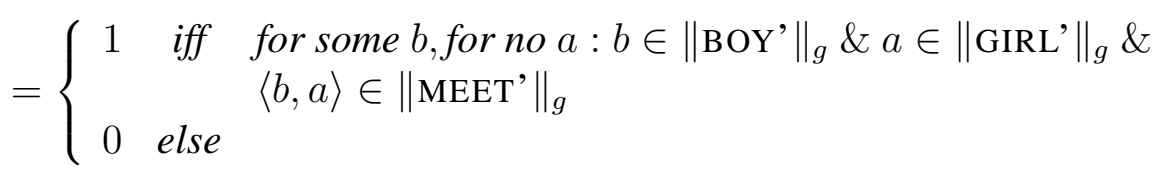

This corresponds to the classical

$$
\exists x \neg \exists y \cdot \operatorname{BOY}^{\prime}(x) \wedge \operatorname{GIRL}^{\prime}(y) \wedge \operatorname{MEET}^{\prime}(x, y)
$$


The restriction of the partial variable $x$ remains within the scope of the first quantifier ccommanding it, and this is $\exists y$ here. This is a configuration that is similar to the Donald Duck problem discussed earlier. The formula in (57) is true iff there is an object with the property that if it is a boy, it met no girl. Of course these are not the truth conditions of (53).

An adequate semantics for quantification should turn those definedness conditions that belong to the bound variable into truth conditions, while the restrictions belonging to other variables should be passed on as definedness conditions.

To implement this intuition, I will use a technique that is inspired by Reinhart (1997). Reinhart assumes that an indefinite with an empty restriction does refer to some special object with non-classical properties. In Reinhart's account, this special object has no properties, so to speak. I will also assume a special object as the referent of such indefinites, but one that is hyperconsistent and has all properties. Let us call this object " $\perp$ ". It is not an element of the universe of discourse, so it does not occur in the extension of non-logical predicates. It is a licit referent of a variable though. If $g(x)=\perp$, then the partial variable $[x \mid \varphi]$ will refer to $\perp$ under $g$, no matter whether or not the restriction is true. This means that there is always an assignment function $g$ that fulfills all restrictions on $x$, namely the one that maps $x$ to $\perp$, and the same holds for all variables. This enables us to keep definedness conditions apart that originate from different variables.

So if the condition $\varphi$ is true, the referent of $[x \mid \varphi]$ under $g$ is $g(x)$. If $\varphi$ is false, $g(x)$ is only defined if it is $\perp$. There is a third option $-\varphi$ may be undefined because some variable different from $x$ occurring in it has an unfulfilled definedness condition. In this case, $[x \mid \varphi]$ is undefined under $g$ even if $g(x)=\perp$.

The modified semantics for first order logic with partial variables is now as follows.

\section{Definition 6 (Semantics of partial variables (final version))}

$$
\|[x \mid \varphi]\|_{g}=\left\{\begin{array}{l}
g(x) \quad \text { if }\|\varphi\|_{g^{x}}=1 \\
\perp \quad \text { if }\|\varphi\|_{g^{x}}=0 \text { and } g(x)=\perp \\
\text { undefined else }
\end{array}\right.
$$

Quantification turns definedness conditions into truth conditions, but it should do so in a discriminating way. The quantifiers should turn all those definedness conditions into truth conditions that are due to restrictions on $x$, while other definedness conditions remain definedness conditions. Suppose the interpretation of $\varphi$ is undefined under $g$. If this undefinedness is due to a violation of a restriction on $x$, then $\varphi$ should be defined under the assignment $g[x / \perp]\{x\}$ because $\perp$ fulfills all restrictions, even inconsistent ones. If, on the other hand, the restrictions on other variables are violated as well, the formula is also undefined under the revised assignment function.

Furthermore, for an existentially quantified formula to be true requires the existence of an object that verifies the formula in question, and likewise for a universally quantified formula to be false. Here we only care about proper, consistent objects, not about $\perp$. Hence quantification should range over the universe without $\perp$. This leads to the final version of the semantics for quantification: 


\section{Definition 7 (Semantics of Quantification (final version))}

$$
\begin{aligned}
& \|\exists x \varphi\|_{g}=\left\{\begin{array}{l}
1 \quad \text { if for some } a \neq \perp:\|\varphi\|_{g[a / x]\{x\}}=1 \\
0 \quad \text { if }\|\varphi\|_{g[x / \perp]\{x\}} \text { is defined, and for all } a \neq \perp:\|\varphi\|_{g[a / x]\{x\}} \neq 1 \\
\text { undefined else }
\end{array}\right. \\
& \|\forall x \varphi\|_{g}=\left\{\begin{array}{l}
1 \quad \text { if }\|\varphi\|_{g[x / \perp]\{x\}} \text { is defined, and for all } a \neq \perp:\|\varphi\|_{g[a / x]\{x\}} \neq 0 \\
0 \quad \text { if for some } a \neq \perp:\|\varphi\|_{g[a / x]\{x\}}=0 \\
\text { undefined else }
\end{array}\right.
\end{aligned}
$$

Note that this revised definition re-establishes the classical equivalence between $\forall x \varphi$ and $\neg \exists x \neg \varphi$.

Let us see what the new interpretation rule has to say about the previous example. Instead of (55) we have the revised interpretation:

$$
\begin{aligned}
& \| \operatorname{MEET}{ }^{\prime}\left(\left[x \mid \text { BOY }^{\prime}(x)\right],\left[y \mid \operatorname{GIRL}^{\prime}(y)\right]\right) \|_{g} \\
& =\left\{\begin{aligned}
1 \text { iff } & g(x) \in\left\|\mathrm{BOY}^{\prime}\right\| \cup\{\perp\} \& g(y) \in\left\|\mathrm{GIRL}^{\prime}\right\| \cup\{\perp\} \& \\
& \langle g(x), g(y)\rangle \in\|\mathrm{MEET},\| \\
0 \quad \text { iff } \quad & g(x) \in\left\|\mathrm{BOY}^{\prime}\right\| \cup\{\perp\} \& g(y) \in\left\|\mathrm{GIRL}^{\prime}\right\| \cup\{\perp\} \& \\
& \langle g(x), g(y)\rangle \notin^{\prime}\left\|\mathrm{MEET}^{\prime}\right\| \\
\text { undefined else } &
\end{aligned}\right.
\end{aligned}
$$

After applying existential quantification once, we have:

$$
\begin{aligned}
& \| \exists y \operatorname{MEET}^{\prime}\left(\left[x \mid \text { BOY }^{\prime}(x)\right],\left[y \mid \operatorname{GIRL}^{\prime}(y)\right]\right) \|_{g} \\
& =\left\{\begin{array}{c}
1 \quad \text { iff } \quad g(x) \in\left\|\mathrm{BOY}^{\prime}\right\| \cup\{\perp\} \& \\
\text { there is an } a \in\|\mathrm{GIRL}\|:\langle g(x), a\rangle \in\left\|\mathrm{MEET}^{\prime}\right\| \\
0 \quad \text { iff } \quad g(x) \in\left\|\mathrm{BOY}^{\prime}\right\| \cup\{\perp\} \& \\
\text { for no } a \in\left\|\mathrm{GIRL}^{\prime}\right\|:\langle g(x), a\rangle \in\left\|\mathrm{MEET}^{\prime}\right\| \\
\text { undefined else }
\end{array}\right.
\end{aligned}
$$

Negation just switches truth values. Existentially quantifying over $x$ then gives us

$$
\begin{aligned}
& \| \exists x \neg \exists y \operatorname{MEET}^{\prime}\left(\left[x \mid \text { BOY }^{\prime}(x)\right],\left[y \mid \operatorname{GIRL}^{\prime}(y)\right]\right) \|_{g}
\end{aligned}
$$

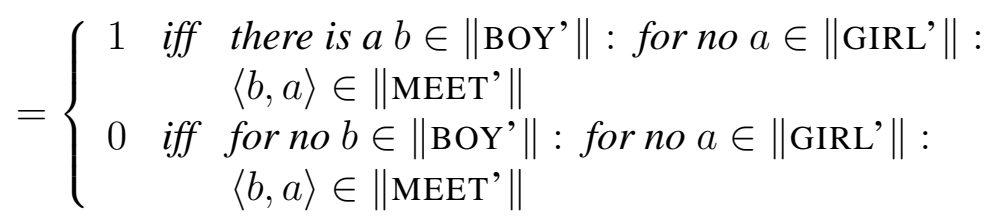

These are in fact the (bivalent) truth conditions of the classical formula

$$
\exists x . \operatorname{BOY}^{\prime}(x) \wedge \neg \exists y . \operatorname{GIRL}^{\prime}(y) \wedge \operatorname{MEET}^{\prime}(x, y)
$$




\subsection{Variable free semantics?}

The final semantics for first order logic with partial variables we end up with turns out to be rather complex. We have to enrich the domain of interpretation with an abstract, inconsistent object, and we employ assignment function that range over sequences of variables rather than over single variables. One might wonder whether this degree of complexity is really necessary.

This touches the issue what the status of variables in natural language semantics actually is. Some researchers, especially categorial grammarians, have argued that natural languages does not use variables. Rather, variables are a convenient technical device that can ultimately be dispensed with, just like logical representations in a surface compositional model of grammar (see especially the discussion in Szabolcsi (1988) and Jacobson (1999)). The technical devices that I introduced in the last two subsections are arguably tailored to solve problems that a variable free system does not have in the first place.

In a variable free system, variable occurrences are replaced by identity functions that function compose with their environment. Having several occurrences of the same variable amounts to merging the argument roles that correspond to them. There is no real counterpart to first order quantification in variable free system. Rather, quantification is dealt with as in Generalized Quantifier theory, i.e. quantifiers are higher order functions that apply to other functions.

There is a natural variable free counterpart to partial variables, namely partial functions. A variable free version of the present theory using partial functions is in fact possible, see Jäger (2001a,b). In this system, the meaning of for instance an indefinite like $a$ boy would just be the identity function over the set of boys.

The improper object $\perp$ was introduced to make sure that the definedness conditions of different variables can be kept apart. In the variable free system, this is automatically the case. Take the example $a$ boy meets a girl. This would be interpreted as a partial function that is defined only over the set of boys. Each boy $b$ is mapped to a partial function $f_{b}$. $f_{b}$ is a partial function that is defined only over the set of girls, and where defined, it maps each girl $g$ to the truth value of $b$ met $g$. Now if there are no boys, the clause denotes the empty function, and if there are boys but no girls, it denotes a constant function on the set of boys that always has the empty function as a value. These are different objects, and there is no problem keeping them apart. The treatment of pronouns inside the description of an indefinite (as discussed in the last subsection) is more intricate in the variable free system, and I will not discuss it here. Suffice it to say that the bound pronoun problem does not arise either due to the combinatorics of the system.

A proponent of variable free semantics could thus make a case that the complexity introduced in the last two subsections is an artifact of the usage of variables, which should be avoided anyway. At the present point, I remain agnostic as to whether the true theory of natural language semantics actually needs variables. It is undeniable though that working with variables makes life a lot easier because it allows to hide a good deal of the semantics complexity behind a convenient notation. (Even fervent proponents of variable free semantics work with the $\lambda$ calculus as representation language instead of the variable free combinatory logic, for exactly this reason.) If the variable free view is correct, there is still nothing wrong with using variables as long as one knows how to get rid of them, and the complexity introduced here is just the price one has to pay for a convenient notation. If, on the other hand, variables are really inevitable, the complexity is 
probably genuine.

\section{Partial variables and presuppositions}

The analysis of indefinites as partial variables is obviously strongly related to Heim's treatment, the only difference being that the descriptive part of an indefinite is part of the definedness conditions rather than of the truth conditions. However, the theory developed here can also be considered a variant of the choice function approach. An indefinite DP like a cup is analyzed as $[x \mid$ CUP' $(x)]$, i.e. as an expression of type $e$. If the NP part cup is analyzed as a predicate of type $\langle e, t\rangle$ like CUP', the indefinite determiner must have the interpretation $\lambda P[x \mid P(x)]$, i.e. a function of type $\langle\langle e, t\rangle, e\rangle$. Furthermore, this function, if defined, always maps a set to one of its members, i.e. it is a choice function. Unlike classical choice functions, this function is undefined for certain sets, and where defined, it always returns the same value, namely $g(x)$, where $g$ is the local assignment function. So the present theory can be presented as the variant of the choice function approach that results if only partial and constant choice functions are considered.

Last but not least, there is a strong affinity between partial variables and presuppositions. Even stronger, I suggest that presuppositions should generally be analyzed as restrictions on partial variables.

Presupposition projection works due to the following equivalence under this account:

Theorem 1 If $[x \mid \psi]$ is a free occurrences of $x$ in $\varphi$ and does not occur in the restriction of a variable, then for all assignments $\mathrm{g}$ :

$$
\|\exists x \varphi([x \mid \psi])\|_{g}=\|\exists x . \varphi(x) \wedge \psi\|_{g}
$$

Proof: See Appendix.

This law accounts for both varieties of presupposition projection, binding and accommodation. Since Karttunen (1974) it is known that at least in the following two configurations, presuppositions are bound (or, in Karttunen's terminology, "filtered"). I use a simple definite description as an example of a presupposition trigger and only consider the existential presupposition triggered by it for the purpose of illustration. The sentence

The cup broke.

triggers the presupposition that there is a cup. However, in the following complex constructions, this presupposition is filtered/bound.

\section{a. Conjunction}

First a cup moved, and then the cup broke.

b. Conditional

If a cup moved, then the cup broke. 
I treat the definite description the cup analogously to its indefinite counterpart, except that it does not have to obey the Novelty Condition, and hence it may (and in our examples indeed does) pick up a familiar variable. Given this and the variable binding conventions of DRT, (63a) and (b) are to be translated as (64a) and (b) respectively.

$$
\begin{array}{ll}
\text { a. } & \exists x \cdot \operatorname{MOVE}^{\prime}\left(\left[x \mid \operatorname{CUP}^{\prime}(x)\right]\right) \wedge \operatorname{BREAK}^{\prime}\left(\left[x \mid \operatorname{CUP}^{\prime}(x)\right]\right) \\
\text { b. } \quad \forall x \cdot \operatorname{MOVE}^{\prime}\left(\left[x \mid \operatorname{CUP}^{\prime}(x)\right]\right) \rightarrow \operatorname{BREAK}^{\prime}\left(\left[x \mid \operatorname{CUP}^{\prime}(x)\right]\right)
\end{array}
$$

Using Theorem 1 twice (and in the case of (b) the inter-definability of the quantifiers), these formulas can be re-written as the equivalent

$$
\begin{aligned}
& \text { a. } \quad \exists x \cdot \operatorname{CUP}^{\prime}(x) \wedge \operatorname{MOVE}^{\prime}(x) \wedge \operatorname{BREAK}^{\prime}(x) \\
& \text { b. } \quad \forall x \cdot \operatorname{CUP}^{\prime}(x) \wedge \operatorname{MOVE}^{\prime}(x) \rightarrow \operatorname{BREAK}^{\prime}(x)
\end{aligned}
$$

Neither formula presupposes the existence of cups. (The first one entails it, but its negation does not.)

More generally, it is an immediate consequence of Theorem 1 that

- If $\varphi \models \psi$, then

$$
\begin{aligned}
& -\exists x . \varphi \wedge \chi([x \mid \psi]) \Longleftrightarrow \exists x . \varphi \wedge \chi(x) \\
& -\forall x . \varphi \rightarrow \chi([x \mid \psi]) \Longleftrightarrow \forall x . \varphi \rightarrow \chi(x)
\end{aligned}
$$

So presupposition binding from the first into the second conjunct, and from the restrictor into the scope of a conditional is correctly predicted to be possible.

Technically, presupposition binding means that the variable that hosts the presupposition is familiar in its local context. As van der Sandt's theory (but unlike other dynamic theories of presuppositions), the present account does not require that a bound presupposition be entailed by its local context. This is empirically justified. In the following example, the presupposition that there is a funny old man, which is triggered by the definite in the second conjunct, is not entailed by its antecedent the teacher, but still binding is possible.

(66) $[\text { The teacher }]_{i}$ entered the room, and the students welcomed [the funny old man $]_{i}$.

Presupposition accommodation amounts to a configuration where the variable hosting the presupposition is novel. Recall that free variables can be bound by applying existential closure at some super-ordinate sentential level (or at the text level). This is tantamount to accommodating the restriction of the variable at this level. Given this, Theorem 1 says that presuppositions can project out of arbitrary embedded contexts. In Karttunen's terminology, this amounts to the claim that every context is a potential hole for presupposition projection. Filters are binding configurations-following van der Sandt, I assume that binding is ceteris paribus preferred over accommodation. So structurally the example (63b) is ambiguous between binding and accommodation, depending on whether a cup and the cup are translated as the same or as different variables. The translation corresponding to the accommodation configuration would be 


$$
\forall x . \operatorname{MOVE}^{\prime}\left(\left[x \mid \operatorname{CUP}^{\prime}(x)\right]\right) \rightarrow \operatorname{BREAK}^{\prime}\left(\left[y \mid \operatorname{CUP}^{\prime}(y)\right]\right)
$$

Here the variable $y$ is free and has to be bound via existential closure. This can be done either at the level of the succedent clause of the conditional or at the matrix level. Since global accommodation is preferred over local accommodation, the resolved translation is

$$
\exists y \forall x . \operatorname{MOVE}^{\prime}\left(\left[x \mid \mathrm{CUP}^{\prime}(x)\right]\right) \rightarrow \text { BREAK}^{\prime}\left(\left[y \mid \mathrm{CUP}^{\prime}(y)\right]\right)
$$

Due to Theorem 1 and the laws of first order logic, this is equivalent to

$$
\exists y \cdot \operatorname{CUP}^{\prime}(y) \wedge \exists x\left(\operatorname{CUP}^{\prime}(x) \wedge \operatorname{MOVE}^{\prime}(x)\right) \rightarrow \operatorname{BREAK}^{\prime}(y)
$$

Since binding is preferred over accommodation, (69) does not represent a pragmatically possible reading of (63b).

Specific indefinites are ordinary presupposition triggers, except for the fact that, as indefinites, they must obey the Novelty Condition. Therefore presupposition binding is not possible here, and accommodation is the only option.

The natural question to ask is whether all presuppositions can be represented as restrictions of partial variables in a natural way. A detailed answer to this question requires further research; I'll just add some remarks on it here. Formally, it is always possible to bury a presupposition in a tautological formula, something of the form

$$
x=[x \mid \varphi]
$$

which has the only effect of introducing the presupposition $\varphi$. While this is technically possible, it is rather ad hoc, and one might wonder whether a linguistically more meaningful approach is possible. I assume that many presuppositions that cannot naturally be analyzed as restrictions on individual variables (like the presuppositions induced by definite descriptions) should be considered restrictions on event variables. Below are some tentative proposals for a representation of the presuppositions induced by aspectual verbs, temporal adverbs, and clefts.

(70) a. Peter quit smoking.

b. QUIT_SMOKING' $($ PETER', $e) \wedge\left[e^{\prime} \mid\right.$ SMOKE' $^{\prime}\left(\right.$ PETER' $\left.\left.^{\prime}, e^{\prime}\right)\right]<e$

(71) a. Peter is tired again.

b. TIRED' (PETER', $e) \wedge\left[e^{\prime} \mid\right.$ TIRED' $\left(\right.$ PETER', $\left.\left.e^{\prime}\right)\right]<e$

(72) a. It was Bill who talked.

b. TALK' (BILL', $\left[e \mid \exists x \cdot\right.$ TALK' $\left.\left.^{\prime}(x, e)\right]\right)$

This approach to the analysis of presuppositions is strongly inspired by van der Sandt's theory, and the empirical predictions are identical. The difference is a conceptual one: van der Sandt assumes two layers of representation between syntax and model-theoretic interpretation, 
the underspecified DRS containing unresolved presuppositions, and the resolved DRS. The analysis of presuppositions as partial variables only requires one intermediate level. Presupposition resolution is part of the mapping from syntax to DRSs (or from S-structure to LF, if you like), alongside with quantifier scope assignment and similar disambiguations. It is in fact possible to reformulate the present theory in an entirely surface compositional way if existential closure is treated as a free semantic operation without syntactic counterpart. Since this technical exercise is of minor importance for the issues discussed in this paper, I refrain from spelling this out here.

\section{Conclusion}

In this paper I introduced and motivated an extension of classical first order logic by the construct of partial variables, i.e. variables that only refer if a certain side condition is fulfilled. Quantification was modified such that it turns definedness conditions of the bound variable into a part of the truth conditions. I tried to argue that presuppositions in natural language should be analyzed as definedness conditions on variables. Furthermore, I adopted the idea from the literature that specificity is a special case of presupposition accommodation. So specific indefinites should be translated as partial variables in the semantic representation. I argued that this approach incorporates insights from the DRT approach and the choice function approach to the semantics of indefinites but avoids the empirical pitfalls of these theories.

The proposed innovation has consequences for a variety of issues in natural language semantics, and the discussion in this article has necessarily been sketchy at times. There are several issues that invite for further research, both empirical and conceptual ones:

- Non-specific indefinites: I deliberately remained neutral about whether non-specific indefinites should be analyzed as partial variables as well. Technically this would predict the correct truth-conditions if local accommodation is applied. However, local accommodation is generally the least preferred option, while the non-specific reading of indefinites is usually much easier to get than the specific one. Furthermore, some indefinites like bare plurals only have a non-specific reading. These arguments seem to suggest that we are dealing with a real ambiguity between specific and non-specific indefinites (as Fodor and Sag (1982) and Kratzer (1998) also suggest). This is of course pure stipulation, and the relation between the different brands of indefinites requires further investigation.

- Presuppositions: I conjectured that all presuppositions can be considered as restrictions on variables. This was only illustrated with few examples though, where the assumption were rather natural. It remains to be shown that this treatment extends to all classes of presupposition triggers.

- Plural: The interaction of plural semantics with partiality requires further scrutiny. The ability of a plural indefinite to receive a specific interpretation partially depends on whether it is exhaustive and distributive. It remains to be seen whether these restrictions can be accommodated into the present theory in a natural way. 
- Quantification: Phillipe Schlenker (p.c.) suggested to me that partial variables lead to an arguably simpler representation of quantificational structures. A universal quantification as in (73a) could for instance be represented as (b):
a. Every man is mortal.
b. $\forall x$ MORTAL' $\left(\left[x \mid \operatorname{MAN}^{\prime}(x)\right]\right)$

An extension of the present system to generalized quantifiers is straightforward, and the restrictive clause of quantifiers can generally be represented as a restriction of the variable that is bound by the quantifier. This observation might have repercussions for the theory of the syntax-semantics interface.

- Compositionality: As pointed out above, treating presuppositions as restrictions on variables does away with one independent level of syntactic representation of van der Sandt's DRT approach to presuppositions, namely the level of unresolved DRSs. The literature contains several proposals for a compositional reformulation of DRT (see for instance Zeevat (1989) and Asher (1993)). To develop a fully surface compositional theory of specificity and presuppositions, such a compositional DRT must be modified such that accommodation, i.e. selective existential closure, can be incorporated. If successful, one of the main arguments against the DRT approach to presuppositions-its non-compositional architecture-would be invalidated.

- Last but not least it is interesting whether the extension of first order logic with partial variables changes its meta-logical properties, and the same question arises for extension thereof like type theory with partial variables. At the present time it is entirely open whether partial variables are just syntactic sugar from a logical point of view or something of higher significance.

\section{Appendix}

Proof of Theorem 1: We need an auxiliary lemma to carry out the proof:

Lemma 1 For all assignments $g$ and expressions $\alpha$, if $[x \mid \varphi]$ is a free occurrence of $x$ in $\alpha$ and does not occur inside the restriction of a variable, then

$$
\|\alpha([x \mid \varphi])\|_{g}=\left\{\begin{array}{l}
\|\alpha(x)\|_{g} \text { if }\|\varphi\|_{g^{x}}=1 \\
\|\alpha(x)\|_{g} \text { if }\|\varphi\|_{g^{x}}=0 \text { and } g(x)=\perp \\
\text { undefined else }
\end{array}\right.
$$

We prove this via induction over the structure of $\alpha$.

- If $\alpha(x)$ is $x$, the conclusion follows directly from the semantics of partial variables.

- Suppose $\alpha(x)$ is $\exists y \psi(x)$. Then $y \neq x$ because $x$ is free in $\alpha$ by assumption. We distinguish four cases: 
1. $\|\varphi\|_{g^{x}}=1$. It follows directly from the definitions that $g^{x}=g[y / a]\{y\}^{x}$ for arbitrary $a$ (including $\perp$ ). Hence by induction hypothesis,

$$
\|\psi(x)\|_{g[y / a]\{y\}}=\|\psi([x \mid \varphi])\|_{g[y / a]\{y\}}
$$

Therefore

$$
\|\psi(x)\|_{g[y / \perp]\{y\}} \text { is defined iff }\|\psi([x \mid \varphi])\|_{g[y / \perp]\{y\}} \text { is defined, }
$$

and this entails that

$$
\|\exists y \psi(x)\|_{g} \text { is defined iff }\|\exists y \psi([x \mid \varphi])\|_{g} \text { is defined. }
$$

Given this, it follows that

$$
\exists a \neq \perp:\|\psi(x)\|_{g[y / a]\{y\}}=1 \text { iff } \exists a \neq \perp:\|\psi([x \mid \varphi])\|_{g[y / a]\{y\}}=1
$$

and hence

$$
\|\exists y \psi(x)\|_{g}=\|\exists y \psi([x \mid \varphi])\|_{g}
$$

2. $\|\varphi\|_{g^{x}}=0$ and $g(x)=\perp$. Observe that $g(x)=g[y / a]\{y\}(x)$ and $g^{x}=g[y / a]\{y\}^{x}$ for arbitrary $a$. Thus by the same reasoning as in the previous case,

$$
\|\exists y \psi(x)\|_{g}=\|\exists y \psi([x \mid \varphi])\|_{g}
$$

3. $\|\varphi\|_{g^{x}}=0$ and $g(x) \neq \perp$. As in the previous case, we make use of the fact that $g(x)=g[y / a]\{y\}(x)$ and $g^{x}=g[y / a]\{y\}^{x}$ for arbitrary $a$. Hence by induction hypothesis, $\|\psi([x \mid \varphi])\|_{g[y / \perp]}\{y\}$ is undefined, and thus $\|\exists y \psi([x \mid \varphi])\|_{g}$ is undefined as well.

4. $\|\varphi\|_{g^{x}}$ is undefined. Since $g^{x}=g[y / \perp]\{y\}^{x},\|\psi([x \mid \varphi])\|_{g[y / \perp]\{y\}}$ is undefined, and thus $\|\exists y \psi([x \mid \varphi])\|_{g}$ is undefined as well.

- By assumption, $\alpha(x)$ cannot have the form $[y \mid \psi(x)]$ because $x$ is assumed not to occur in the restriction of another variable.

- $\alpha(x)$ has the form $\psi(x) \rightarrow \chi, \chi \rightarrow \psi(x)$, or $\neg \psi(x)$. Suppose $\|\varphi\|_{g^{x}}=1$, or $\|\varphi\|_{g^{x}}=0$ and $g(x)=\perp$. Then by induction hypothesis, $\|\psi(x)\|_{g}=\|\psi([x \mid \varphi])\|_{g}$. It follows from the semantics of the propositional connectives that then $\|\alpha(x)\|_{g}=\|\alpha([x \mid \psi])\|_{g}$. If, on the other hand, $\|\varphi\|_{g^{x}}=0$ and $g(x) \neq \perp$, or $\|\varphi\|_{g^{x}}$ is undefined, then $\|\psi([x \mid \varphi])\|_{g}$ is undefined, and thus $\|\alpha([x \mid \varphi])\|_{g}$ is undefined as well.

- All other connectives can be defined in terms of $\neg, \rightarrow$, and $\exists$. 
This completes the proof of lemma 1. I proceed to the proof of theorem 1.

- Suppose $\|\exists \varphi([x \mid \psi])\|_{g}=1$. Then there is an $a \neq \perp$ such that $\|\varphi([x \mid \psi])\|_{g[x / a]\{x\}}=1$. Since $g[x / a]\{x\}(x)=a \neq \perp$, it follows from lemma 1 that $\|\varphi(x)\|_{g[x / a]\{x\}}=1$ and $\|\psi\|_{g[x / a]\{x\}^{x}}=1$. By the definitions, $g[x / a]\{x\}^{x}=g[x / a]\{x\}$, thus $\|\psi\|_{g[x / a]\{x\}}=1$, and therefore $\|\varphi(x) \wedge \psi\|_{g[x / a]\{x\}}=1$. From the semantics of existential quantification, we conclude that $\|\exists x . \varphi(x) \wedge \psi\|_{g}=1$.

- Suppose $\|\exists x . \varphi(x) \wedge \psi\|_{g}=1$. By the semantics of $\exists$, there is an $a \neq \perp$ such that $\|\varphi(x) \wedge \psi\|_{g[x / a]\{x\}}=1$. Hence $\|\varphi(x)\|_{g[x / a]\{x\}}=1$ and $\|\psi\|_{g[x / a]\{x\}}=1$. Since $g[x / a]\{x\}=g[x / a]\{x\}^{x},\|\psi\|_{g[x / a]\{x\}^{x}}=1$. By lemma 1, $\|\varphi([x \mid \psi])\|_{g[x / a]\{x\}}=1$, and by the semantics of $\exists,\|\exists \varphi([x \mid \psi])\|_{g}=1$.

- Suppose $\|\exists \varphi([x \mid \psi])\|_{g}=0$. Then $\|\varphi([x \mid \psi])\|_{g[x / \perp]\{x\}}$ is defined, and there is no $a \neq \perp$ with $\|\varphi([x \mid \psi])\|_{g[x / a]\{x\}}=1$. By lemma 1, $\|\varphi(x)\|_{g[x / \perp]\{x\}}$ and $\|\psi\|_{g[x / \perp]\{x\}^{x}}$ are defined. Since $g[x / \perp]\{x\}^{x}=g[x / \perp]\{x\},\|\psi\|_{g[x / \perp]\{x\}}$ is defined, hence $\|\varphi(x) \wedge \psi\|_{g[x / \perp]\{x\}}$ is defined as well. Now suppose there were a $b \neq \perp$ with $\|\varphi(x) \wedge \psi\|_{g[x / b]\{x\}}=1$. Then we would have $\|\varphi(x)\|_{g[x / b]\{x\}}=1$ and $\|\psi\|_{g[x / b]\{x\}}=1$ as well. Since $g[x / b]\{x\}=$ $g[x / b]\{x\}^{x}$, we would get $\|\psi\|_{g[x / b]\{x\}^{x}}=1$, and thus by lemma $1,\|\varphi([x \mid \psi])\|_{g[x / b]\{x\}}=$ 1 , contra assumption. Hence there is no $b \neq \perp$ with $\|\varphi(x) \wedge \psi\|_{g[x / b]\{x\}}=1$, and therefore $\|\exists x . \varphi(x) \wedge \psi\|_{g}=0$.

- Finally, suppose $\|\exists x . \varphi(x) \wedge \psi\|_{g}=0$. Then $\|\varphi(x) \wedge \psi\|_{g[x / \perp]\{x\}}$ is defined, and thus both $\|\varphi(x)\|_{g[x / \perp]\{x\}}$ and $\|\psi\|_{g[x / \perp]\{x\}}$ are defined. By lemma $1,\|\varphi([x \mid \psi])\|_{g[x / \perp]\{x\}}$ is also defined. It also follows from the assumption that there is no $a \neq \perp$ with $\| \varphi(x) \wedge$ $\psi \|_{g[x / a]\{x\}}=1$. Now suppose there were a $b \neq \perp$ with $\|\varphi([x \mid \psi])\|_{g[x / b]\{x\}}=1$. By lemma 1, it would follow that $\|\varphi(x)\|_{g[x / b]\{x\}}=1$ and $\|\psi\|_{g[x / b]\{x\} 1}=1$. Since $g[x / b]\{x\}=g[x / b]\{x\}^{x},\|\varphi(x)\|_{g[x / b]\{x\}^{x}}=1$. Therefore $\|\varphi(x) \wedge \psi\|_{g[x / b]\{x\}}=1$, contra assumption. It follows that there is no $b \neq \perp$ with $\|\varphi([x \mid \psi])\|_{g[x / b]\{x\}}=1$. We hence have $\|\exists x \varphi([x \mid \psi])\|_{g}=0$.

This completes the proof.

\section{Author's address}

Gerhard Jäger

University of Potsdam, Institute for Linguistics

PF 601553

D-14415 Potsdam, Germany

jaegerdling.uni-potsdam.de

January 1 - June 30, 2004:

Stanford University, Dept. of Linguistics, Building 460, Stanford, CA 94305-2150, USA 


\section{Acknowledgment}

First of all I would like to thank Cornelia Endriss, both for getting me interested in the issue of specificity and for ongoing discussions on the subject. Furthermore I thank Philippe Schlenker, Christian Ebert, Yael Sharvit, Peter Staudacher, and the audiences of related talks at the University of Chicago, the University of Konstanz and the ZAS Berlin for important feedback.

\section{References}

Abusch, Dorit (1994); 'The scope of Indefinites'. Natural Language Semantics 2:83-135. Asher, Nicholas (1993); Reference to Abstract Objects in Discourse. Kluwer, Dordrecht.

Beaver, David (2001); Presupposition and Assertion in Dynamic Semantics. CSLI Publications, Stanford.

Chierchia, Gennaro (2001); 'A Puzzle About Indefinites'. In Carlo Cecchetto, Gennaro Chierchia, and Maria Teresa Guasti (eds.), Semantic Interfaces, CSLI Publications, Stanford.

Cresti, Diana (1995); Indefinite Topics. Ph.D. thesis, MIT.

Dekker, Paul (2002); 'A Pragmatic View upon Indefinites'. In Klaus von Heusinger, Ruth Kempson, and Wilfried Meyer-Viol (eds.), Proceedings of the ESSLLI 13 Workshop on Choice Functions and Natural Language Semantics, Fachbereich Sprachwissenschaft der Universität Konstanz, Arbeitspapier Nr. 110, 17-34.

Diesing, Molly (1992); Indefinites. MIT Press, Cambridge (Mass.).

Endriss, Cornelia (2001); The Double Scope of Quantifier Phrases. Master's thesis, University of Potsdam.

Farkas, Donka (1981); 'Quantifier Scope and Syntactic Islands'. In Papers from the 17th Regional Meeting of the Chicago Linguistic Society, University of Chicago, 59-66.

Farkas, Donka and Anastasia Giannakidou (1996); 'How Clause-bounded is the Scope of Universals?' In Teresa Galloway and Justin Spence (eds.), Proceedings of SALT VI, CLC Publications, Cornell University, Ithaca, 35-52.

Fodor, Janet and Ivan Sag (1982); 'Referential and Quantificational Indefinites'. Linguistics and Philosophy 5:355-398.

Geurts, Bart (1999a); Presuppositions and Pronouns. Elsevier, London.

Geurts, Bart (1999b); 'Specifics'. In Bart Geurts, Manfred Krifka, and Rob van der Sandt (eds.), Focus and Presupposition in Multi-Speaker Discourse, ESSLLI'99, University of Utrecht, 99129.

Geurts, Bart (2000); 'Indefinites and Choice Functions'. Linguistic Inquiry 31:731-738.

Heim, Irene (1982); The Semantics of Definite and Indefinite Noun Phrases. Ph.D. thesis, University of Massachusetts, Amherst.

Hintikka, Jaakko (1986); 'The semantics of a certain'. Linguistic Inquiry 17:331-336.

Jacobson, Pauline (1999); 'Towards a variable-free semantics'. Linguistics and Philosophy 22:117-184.

Jäger, Gerhard (2001a); Anaphora and Type Logical Grammar. OTS Working Papers. Utrecht Institute of Linguistics (OTS), Utrecht. 
Jäger, Gerhard (2001b); 'Indefinites and Sluicing. A type logical approach'. In Robert van Rooy and Martin Stokhof (eds.), Proceedings of the 13th Amsterdam Colloquium, ILLC, University of Amsterdam, 114-119.

Kamp, Hans and Uwe Reyle (1993); From Discourse to Logic. Introduction to Modeltheoretic Semantics of Natural Language, Formal Logic and Discourse Representation Theory. Kluwer, Dordrecht.

Karttunen, Lauri (1974); 'Presuppositions and Linguistic Context'. Theoretical Linguistics 1:181-94.

Kratzer, Angelika (1998); 'Scope or Pseudscope? Are there Wide Scope Indefinites?' In Susan Rothstein (ed.), Events and Grammar, Kluwer, Dordrecht, 163-196.

Krifka, Manfred (1998); 'Non-novel Indefinites in Adverbial Quantification'. In Carlota Smith (ed.), Workshop Proceedings: Non-narrative Discourse, Texas Linguistic Forum, Department of Linguistics, University of Texas at Austin.

Reinhart, Tanya (1992); 'Wh-in-situ: an apparent paradox'. In Paul Dekker (ed.), Proceedings of the Eighth Amsterdam Colloquium, University of Amsterdam, 483-491.

Reinhart, Tanya (1995); Interface Strategies. OTS Working Papers. Utrecht Institute of Linguistics (OTS), Utrecht.

Reinhart, Tanya (1997); 'Quantifier Scope: How Labor is Divided Between QR and Choice Functions'. Linguistics and Philosophy 20:335-397.

Reniers, Fabien (1997); How to (S)cope with Indefinites. Master's thesis, University of Utrecht. Ruys, Eddy (1992); The scope of indefinites. Ph.D. thesis, University of Utrecht.

Szabolcsi, Anna (1988); Bound Variables in Syntax. (Are there any?). Institute of Linguistics, Hungarian Academy of Sciences, Budapest.

Szabolcsi, Anna (1997); 'Strategies for Scope Taking'. In Anna Szabolcsi (ed.), Ways of Scope Taking, Kluwer, Dordrecht, 109-154.

van der Sandt, Rob (1992); 'Presupposition Projection as Anaphora Resolution'. Journal of Semantics 9:333-377.

van Geenhoven, Veerle (1998); Semantic Incorporation and Indefinite Descriptions. CSLI Publications, Stanford.

Winter, Yoad (1997); 'Choice Functions and the Scopal Semantics of Indefinites'. Linguistics and Philopsophy 20:399-467.

Yeom, Jae-Il (1998); A Presuppositional Analysis of Specific Indefinites. Garland Publishing, New York, London.

Zeevat, Henk (1989); 'A Compositional Approach to Discourse Representation Theory'. Linguistics and Philosophy 12:95-131. 